\title{
Novel Caffeic Acid Nanocarrier: Production, Characterization, and Release Modeling
}

\author{
Milad Fathi, ${ }^{1}$ Maryam Mirlohi, ${ }^{2}$ Jaleh Varshosaz, ${ }^{3}$ and Golnoosh Madani ${ }^{4}$ \\ ${ }^{1}$ Department of Food Science and Technology, Faculty of Agriculture, Isfahan University of Technology (IUT), \\ Isfahan 84156-83111, Iran \\ ${ }^{2}$ Food Security Research Center, School of Nutrition and Food Science, Isfahan University of Medical Science (MUI), \\ Isfahan 8174-73461, Iran \\ ${ }^{3}$ Faculty of Pharmacy, Isfahan University of Medical Science (MUI), Isfahan 81746-73461, Iran \\ ${ }^{4}$ Department of Biotechnology, Faculty of Agriculture, Isfahan University of Technology (IUT), Isfahan 84156-83111, Iran \\ Correspondence should be addressed to Maryam Mirlohi; m_mirlohi@hlth.mui.ac.ir
}

Received 9 June 2013; Revised 9 September 2013; Accepted 10 September 2013

Academic Editor: Krasimir Vasilev

Copyright (c) 2013 Milad Fathi et al. This is an open access article distributed under the Creative Commons Attribution License, which permits unrestricted use, distribution, and reproduction in any medium, provided the original work is properly cited.

\begin{abstract}
This paper deals with the development of novel nanocarriers using layer by layer carbohydrate coating of caffeic acid loaded solid lipid nanoparticles (SLNs) to improve stability and colon delivery of the poorly water-soluble caffeic acid. Three biopolymers (chitosan, alginate, and pectin) in different concentrations $(0.1,0.25$, and $0.5 \%)$ were electrostatically coated over the SLN surface. The size and zeta potential of produced nanocarriers were measured using photon correlation spectroscopy. Mathematical models (i.e., zero-order, first-order, Higuchi, Ritger-Peppas, reciprocal powered time, Weibull, and quadratic models) were used to describe the release and kinetic modeling in gastrointestinal solution (GIS). Also, antioxidant activity of caffeic acid during the release in GIS was investigated using DPPH and reducing activity methods. The prepared treatments coated by alginate-chitosan as well as pectin-chitosan coated SLN at the concentration of $0.1 \%$ showed nanosized bead; the latter efficiently retarded the release of caffeic acid in gastric media up to 2.5 times higher than that of SLN. Zeta potential values of coated samples were found to significantly increase in comparison to SLN indicating the higher stability of produced nanocarriers. Antioxidant activity of caffeic acid after gastric release did not result in the same trend as observed for caffeic acid release from different treatments; however, in line with less caffeic acid release in the intestine solution by the effect of coating, lower antioxidant activity was determined at the end stage of the experiment.
\end{abstract}

\section{Introduction}

Caffeic acid (3,4-dihydroxycinnamic acid) is a natural antioxidant found in various agricultural products such as fruits, vegetables, tea, olive oil, and coffee bean [1]. It acts as an antioxidant by scavenging oxygen-free radicals and chelating prooxidant metal ions [2]. Caffeic acid has also shown antimicrobial properties against Escherichia coli, Bacillus cereus, Staphylococcus aureus, Listeria monocytogenes, and some yeast [3]. However, it suffers from low stability in UV irradiation and oxygen presence $[2,3]$. On the other hand, low aqueous solubility and bitter taste limit its application for food fortification.
Nanoencapsulation of food bioactives possesses different advantages including masking unpleasant taste, possibility of application of low soluble compounds, and protection against unfavorable environmental and processing conditions [4]. It is also supposed that colon delivery of food bioactives, particularly in nanocarriers is favorable due to higher bioavailability and positive effect on natural intestine flora. Solid lipid nanoparticles (SLNs) are new generation of nanocarriers which attract increasing attention due to their advantages such as high encapsulation efficiency, slow degradation rate, and possibility of large scale production [5]. They have been produced and characterized for encapsulating of hesperetin for food fortification. In spite of the different 
benefits, there are some limitations possess to SLN including their initial rapid release (burst release) and their low stability [6]. Therefore, modification of nanocarriers is necessary to overcome these limitations.

Chitosan (poly[ $\beta$-(1-4)-2-amino-2-deoxy-d-glucopyranose]; $\mathrm{CH})$ is the second most abundant polysaccharides in nature after cellulose. There are some valuable characteristics possess to chitosan (e.g., biocompatibility, biodegradability, bioadhesiveness, and antimicrobial properties) which lead to its application for encapsulation of food bioactives $[5,7,8]$. However as a weak base with a $\mathrm{pK}_{a}$ value of the d-glucosamine residue of about $6.2-7.0$, it is soluble in acidic media [9]. Hence, chitosan cannot be considered as a protective biopolymer for encapsulating of bioactives against the gastric media and therefore might not be alone applied for colon delivery.

Alginate $(\mathrm{AL})$ is a water soluble linear anionic polysaccharide formed from alternating blocks of 1-4 linked $\alpha$-Lguluronic and $\beta$-Dmannuronic acid residues. It has been reported to be mucoadhesive, biodegradable, and biocompatible $[10,11]$. Gel formation of AL is induced using crosslinking with divalent cations such as $\mathrm{Ca}^{+2}$. The gelation and cross-linking of the AL are mainly achieved by exchanging of sodium ions from the guluronic acids with the $\mathrm{Ca}^{+2}$ ions, and zipping of guluronic groups to form the egg-box-like conformation [12]. Alginate also shows $\mathrm{pH}$ sensitivity, while in contrast to chitosan, the release of encapsulated bioactive could be postponed in low $\mathrm{pH}$, which is advantageous for colon delivery of acidic sensible bioactive ingredients $[8,13,14]$.

Pectin (PEC) is a linear anionic polysaccharide, mainly consisting of linearly connected $\alpha$ - $(1 \rightarrow 4)$-d-galacturonic acid residues and is considered as a biocompatible, biodegradable, nontoxic, and mechanically resistant against physical shear. Pectin is classified as low methoxyl (LM, with a 25-50\% degree of methoxylation) and high methoxyl (HM, with a $50-80 \%$ degree of methoxylation). LM pectin can also form an egg-box-like conformation gel by ionotropic gelation in presence of divalent $\mathrm{Ca}^{2+}$ ions [15]. Similar to alginate, LM pectin is $\mathrm{pH}$ sensitive and is favorable for food bioactive oral delivery.

Caffeic acid is a sensitive compound against oxidative degradation and also shows poor aqueous solubility and bitter taste. Therefore, its protection is necessary before its application for food fortification. To our knowledge, there is not any effort to enhance stability of caffeic acid. Therefore, the aims of this work were to produce and characterize novel nanocarriers for entrapment of caffeic acid and investigate the effect of encapsulation on caffeic acid antioxidant properties after release in gastrointestinal condition.

\section{Materials and Methods}

2.1. Materials. Caffeic acid was purchased from SigmaAldrich (USA). Chitosan (low molecular weight, MW 150000), sodium alginate, and LM pectin were supplied by Sigma-Aldrich Company (Canada). Glycerol monostearate (GMS; Condea, Germany), glycerol behenate (Compritol 888;
Gattefossé, France), and Tween 80 (Merck, Germany) were applied at analytical grade. All other chemicals and reagents were at least of analytical grade.

\subsection{Methods}

2.2.1. SLN Production. The SLN production method was adjusted based on the procedure mentioned in a previous study [6]. Briefly, the aqueous phase was added to caffeic acid containing melted lipid phase at $80^{\circ} \mathrm{C}$ and stirred in $2000 \mathrm{rpm}$ for $1 \mathrm{~min}$. To form coarse and nanoemulsions the preemulsion was subjected to bath (10 min; Powersonic 505; Hwashin Technology, Gyeonggi-do, Republic of Korea) and probe ( $1 \mathrm{~min}$, in amplitude $50 \%$ and power of $100 \mathrm{~W}$-TT13 probe; Bandelin, Germany, Berlin) sonication, respectively. In order to prevent temperature increase, the probe sonicator was inactive in 2 second intervals. The attained emulsion was cooled down in an ice bath for 30 minutes to recrystallize lipid forming SLN. The encapsulation load and efficiency were determined using centrifugation method [6].

2.2.2. SLN Layer by Layer Coating. Chitosan solution was prepared by dissolving into acetic acid (0.1\%) and stirring overnight. Alginate and pectin were dissolved in distilled water. SLNs were coated layer by layer coating of alginate or pectin with chitosan. A solution of $7.5 \mathrm{~mL}$ chitosan $(0.1 \%)$ was added dropwise into a beaker containing $15 \mathrm{~mL}$ SLN solution under $1000 \mathrm{rpm}$ stirring. Then, $15 \mathrm{~mL}$ of alginate or pectin solution $(0.1,0.25$, and $0.5 \%)$ was dropped into the SLN-chitosan solution. Finally, appropriate amount of $18 \mathrm{mM}$ calcium chloride solution was dropped into resulted solution, the $\mathrm{pH}$ was set on 5.2, and prepared nanoparticles were held with an additional stirring for $30 \mathrm{~min}$ to form uniform particle size. Subsequently, for future characterization, nanoparticles were freeze-dried at $-80^{\circ} \mathrm{C}$ in $0.001 \mathrm{mbar}$ for $48 \mathrm{~h}$ using a freeze-dryer (Christ Alpha LD, Germany).

2.2.3. Particle Size and Zeta Potential. The average particle size and zeta potential were determined by photon electron spectroscopy (PCS) using Zetasizer (NanoSizer 3000, Malvern Instruments, Malvern, UK). The size measurements were performed at a fixed angle of 90 degrees using the volume distribution. The polydispersity index (PDI) measures the size distribution of the nanocarriers. The lower the PDI, the narrower the size distribution. Zeta potential which is an indicator of surface charge was measured based on mean electrostatic mobility applying HelmholtzSmoluchowski equation [16].

2.2.4. Morphology Characterization. Morphological study of the SLN and coated nanoparticles was performed using transmission electron microscopy (TEM). Samples were negatively stained with uranyl acetate $(2 \%)$ and dried on carbon coated grids at room temperature.

2.2.5. Caffeic Acid Release and Kinetic Modeling. Caffeic acid release was studied in gastric and intestinal solutions applying dialysis bag method at $37^{\circ} \mathrm{C}$ and $100 \mathrm{rpm}$. Two milliliters 
caffeic acid loaded SLN or solution of freeze-dried coated samples (with equal amount of caffeic acid) were sealed into dialysis bag (Sigma, Canada) with a $12 \mathrm{kDa}$ cut-off. The bag was then placed into a $40 \mathrm{~mL}$ gastric buffer for $2 \mathrm{~h}$. It was subsequently subjected to the intestinal buffer $(50 \mathrm{~mL})$ for $6 \mathrm{~h}$. At appropriate time intervals, the amount of released caffeic acid was determined spectrophotometrically at wavelength of $295 \mathrm{~nm}$. The release data were kinetically evaluated by zero-order, first-order, Higuchi, Rigter-Peppas, reciprocal powered time, Weibull, and quadratic models (see (1)-(7)) $[6,17,18]$ :

$$
\begin{gathered}
C=K t, \\
C=[1-\exp (-K t)] \times 100, \\
C=K t^{0.5}, \\
C=K t^{n}, \\
\left(\frac{1}{C}-1\right)=\frac{K}{t^{a}}, \\
\ln [-\ln (1-C)]=a_{w} \ln t-\ln b_{w}, \\
C=100\left(K_{1} t^{2}+K_{2} t\right),
\end{gathered}
$$

where $C$ is caffeic acid concentration (\%) at time $t, K$ is kinetic constant, and $n$ is release exponent. The latest is used to characterize different release mechanisms. Encapsulant release from spherical carriers with $0.43 \leq n$ is controlled by Fickian diffusion mechanism, and $n \geq 0.85$ is commanded for dissolution phenomenon, and $0.43<n<0.85$ is governed by combination of two mechanisms [19].

The shape parameter, $a_{w}$, in Weibull model characterizes thecurve as either exponential $\left(a_{w}=1\right)$ (Case 1), sigmoid, Sshaped, with upward curvature followed by a turning point $\left(a_{w}>1\right)$ (Case 2), or parabolic, with a higher initial slope and after that consistent with the exponential $\left(a_{w}<1\right)$ (Case 3) [18].

2.2.6. Reducing Activity Measurement. Either the gastric or intestinal buffers were subjected to reducing activity test as an indicator for antioxidative capability of the released caffeic acid. The method was adopted from Rekha [20]. A test sample or distilled water (control) $(0.5 \mathrm{~mL})$ was added to $0.5 \mathrm{~mL}$ potassium ferricyanide solution $1 \%$ (Merck, Germany) and $0.5 \mathrm{~mL}$ sodium phosphate buffer $(0.02 \mathrm{M}, \mathrm{pH}$ $7)$. The mixture was incubated at $50^{\circ} \mathrm{C}$ for $20 \mathrm{~min}$ and then $0.5 \mathrm{~mL}$ trichloroacetic acid (0.5 mL, Merck, Germany) was added. The mixture was centrifuged at $780 \mathrm{~g}$ for $5 \mathrm{~min}$. The upper layer $(1.5 \mathrm{~mL})$ was mixed with $0.1 \%$ ferri chloride $(0.2 \mathrm{~mL}$, Merck, Germany) and the absorbance was measured at $700 \mathrm{~nm}$. The higher absorbance of the given mixture would present the higher reducing activity. The following equations were used to indicate the concentrations of caffeic acid balanced with the reducing activity of the released caffeic acid.

Reducing activity standard curve in gastric buffer:

$$
x=32.3 y-1.25 \text {. }
$$

Reducing activity standard curve in intestinal buffer:

$$
x=55 y-7.5 \text {. }
$$

2.2.7. DPPH Radical Scavenging Activity. Free radical scavenging activity of the gastric and intestinal solution containing caffeic acid was measured by DPPH method, as described by Shinde et al. with minor modifications [21]. Briefly, $0.2 \mathrm{~mL}$ of each sample was added to $3.8 \mathrm{~mL}$ DPPH ethanol solution (final concentration was adjusted at $0.1 \mathrm{mM}$ ) in a test tube. The mixture was mixed vigorously for $1 \mathrm{~min}$ by vortexing and was left to stand at room temperature in the dark for $30 \mathrm{~min}$. Thereafter, the absorbance of the sample was measured using the UV-visible spectrophotometer at $517 \mathrm{~nm}$ against ethanol blank. A negative control (control) was taken after adding $\mathrm{DPPH}$ solution to $0.2 \mathrm{~mL}$ of the respective gastric of intestinal buffer. The percent of DPPH discoloration of the sample was calculated according to the following equation:

$$
\text { Percentage of discoloration }=\left[1-\left(\frac{A_{\text {sample }}}{A_{\text {control }}}\right)\right] \times 100 \text {. }
$$

The scatter plots of absorbance against different concentration of caffeic acid in gastric and intestinal condition were provided individually, and the following equations were used for each of the media.

$\mathrm{DPPH}$ discoloration standard curve in gastric buffer:

$$
x=126-53.9 y .
$$

DPPH discoloration standard curve in intestinal buffer:

$$
x=182-355 y .
$$

2.2.8. Statistical Analysis. All experiments were performed at least with 3 replications, and the average values were reported. Statistical analyses were carried out using MSTAT software (version C). Data were subjected to analysis of variance (ANOVA), and means were compared using "Duncan" test at $5 \%$ significant level.

\section{Results and Discussion}

SLN, AL-CH, and PEC-CH coated SLNs were produced to enhance stability and functionality of caffeic acid. Encapsulation efficiency and load of produced SLN were 71.21 \pm $1.2 \%$ and $4.73 \pm 0.35 \%$, respectively. It is noteworthy that in a previous paper, it has been indicated that chemical compositions of the materials did not change during layer by layer coating [8]. In the following subsections, some features of developed nanocarriers such as size, zeta potential, 


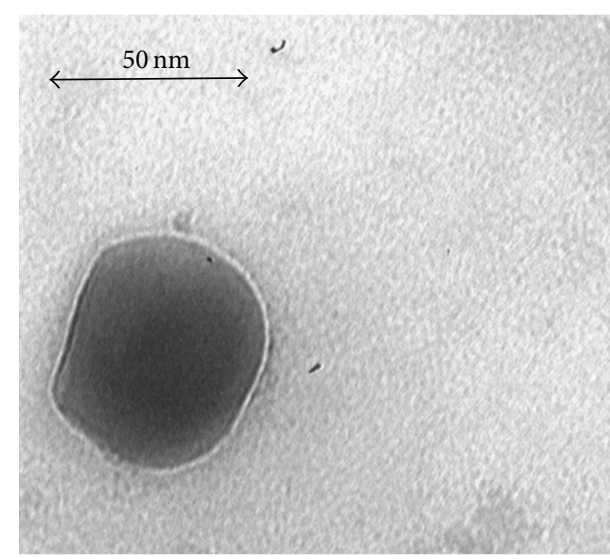

(a)

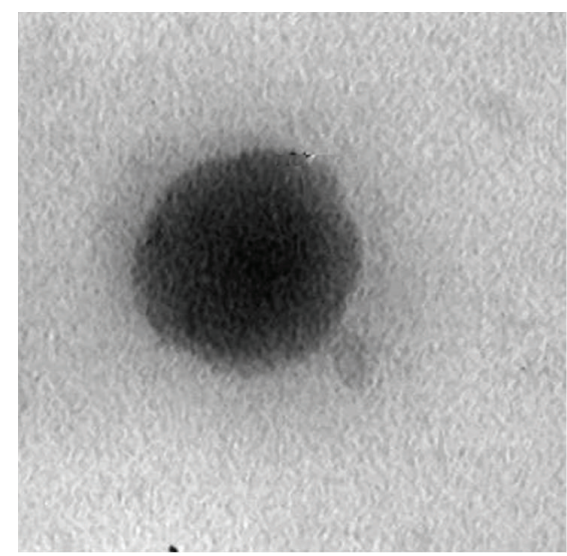

(b)

FIgURE 1: TEM morphology of caffeic acid loaded SLN (b) and PEC-CH coated SLN (a).

morphology, and release behavior are studied; the effect of different treatments on antioxidant activity of caffeic acid is discussed afterwards.

\subsection{Size, Zeta Potential, and Morphological Characterization.} Size, PDI, and zeta potential values of produced nanocarriers were tabulated in Table 1.

As expected, the SLN showed the smallest size $(49.65 \pm 1.12 \mathrm{~nm})$. The developed SLNs are significantly small in comparison to some produced SLN $[6,22]$ and NLC $[23,24]$. The smaller size of the particles leads to their faster movement due to Brownian motion and therefore higher dispersion stability against the gravity. On the other hand, zeta potential value of produced SLN was found to be near zero $(-2.57 \pm 1.03 \mathrm{mV})$. This low zeta potential in comparison to the literature data $[25,26]$ is due to the fact that there is almost the same positive and negative electron charge over the nano carriers' surface. Zeta potential is a crucial factor in nanoparticle stability. The particles with higher zeta potential show bigger repulsion force which leads to higher dispersion stability against aggregation. The low zeta potential of caffeic acid loaded SLN could be attributed to the neutral nature of applied lipid. Therefore, surface coating of nanoparticle using charged biopolymer could enhance their zeta potential. The results indicated that coating lead to increase of size and zeta potential. With the increasing coating concentration, the sizes of nanocarriers significantly $(P<0.05)$ increased in consequence. The negatively charged calcium alginate complex in pregel state can interact with positively charged chitosan [27]. The negative zeta potential of AL-CH coated SLN shows that Ca-AL properly covered the chitosan surface and therefore could be well protected against acidic condition in the gastric medium. Similar results were obtained by Mladenovska research team [28, 29] who showed AL-CH biopolymer had negative zeta potential. The absolute values of zeta potential of AL-CH coated nanoparticles increased by alginate concentration.

PEC-CH coated SLN in $0.1 \%$ pectin concentration showed size of $52.60 \pm 0.31 \mathrm{~nm}$ (Table 1). However, in higher concentrations of pectin, the micron size beads were constructed; therefore, their size and zeta potential were not reported due to the unreliability of the applied instrument. Similar results were obtained in our previous study for hesperetin loaded nanoparticles [8]. The micron size PEC$\mathrm{CH}$ beads were also reported in the literature $[30,31]$. The surface charge of pectin was found to be negative [32]. The positive zeta potential of $0.1 \%$ pectin-chitosan coated nanoparticles might be attributed to low pectin concentration and presence of chitosan on the surface of nanoparticles. Luo et al. produced chitosan coated NLC and observed that the zeta potential of NLC $(-0.45 \mathrm{mV})$ increased by chitosan coating $(+20.70 \mathrm{mV})$ [33].

The PDI values of all produced nanocarriers were found to be lower than 0.5 indicating their narrow size distribution.

The morphological images of SLN and PEC-CH coated nanoparticles obtained from transmission electron microscopy were depicted in Figure 1.

The obtained sized from TEM was in accordance with PCS analysis. Both SLN and coated nanoparticles showed spherical shape. The double shell layer in coated carrier could be obviously observed in TEM image.

3.2. Caffeic Acid Release. Two drawbacks of SLN, namely, low zeta potential and rapid release in gastric media motivated us to produce coated SLN. The release profiles of developed nanocarriers were shown in Figure 2.

Release and absorption of encapsulated food bioactives are supposed to be in intestine for higher bioavailability. More than 55\% of caffeic acid released from SLN during the first two hours in gastric media (Figure 2(a)), and therefore, it was necessary to modify SLN for bioactive protection against gastric condition. AL-CH and PEC$\mathrm{CH}$ coated nanoparticles could both retard caffeic acid release in gastric condition. Caffeic acid releases from AL$\mathrm{CH}$ coated nanoparticles in the first 2 hours were found to be $30.32 \pm 0.08,24.66 \pm 1.00$, and $29.05 \pm 0.99$ for alginate concentrations of $0.1,0.25$, and $0.5 \%$, respectively (Figure 2(b)). No significant difference was observed among 
TABLE 1: Size, PDI, and zeta potential of developed caffeic acid loaded nanocarriers.

\begin{tabular}{lccc}
\hline Nanocarrier & Size $(\mathrm{nm})$ & Polydispersity index $($ PDI $)$ & Zeta potential $(\mathrm{mV})$ \\
\hline SLN & $49.65 \pm 1.12$ & $0.47+0.02$ & $-2.57 \pm 1.03$ \\
\hline AL-CH coated SLN-0.1\% & $74.26 \pm 8.26$ & $0.45 \pm 0.07$ & $-21.87 \pm 3.04$ \\
AL-CH coated SLN-0.25\% & $109 \pm 7.22$ & $0.49 \pm 0.05$ & $-31.60 \pm 2.32$ \\
AL-CH coated SLN-0.5\% & $235.93 \pm 24.1$ & $0.33 \pm 0.03$ & $-35.20 \pm 3.41$ \\
PEC-CH coated SLN-0.1\% & $52.60 \pm 0.31$ & $0.42 \pm 0.01$ & $14.75 \pm 0.35$ \\
\hline
\end{tabular}

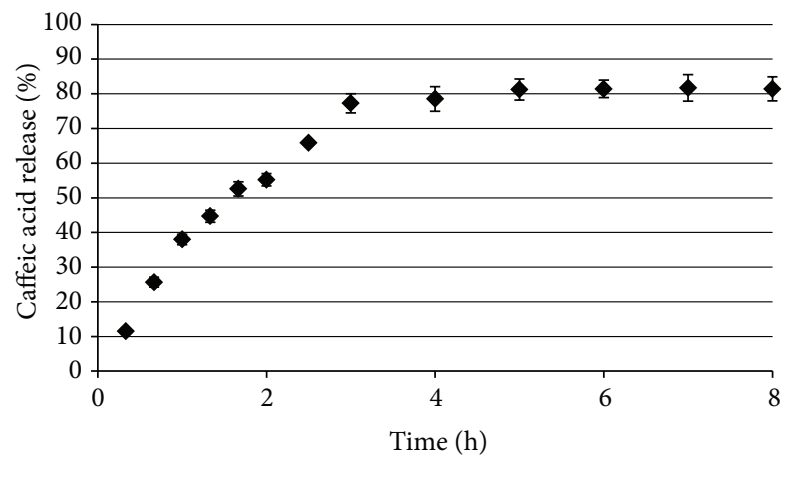

- SLN

(a)

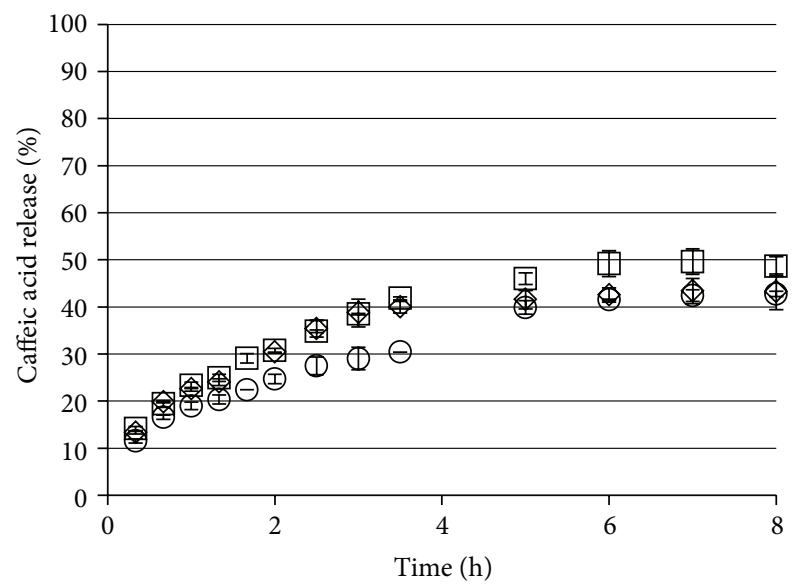

$\diamond \mathrm{AL}-\mathrm{CH}(0.1 \%)$

$\mathrm{OAL}-\mathrm{CH}(0.25 \%)$

$\square \mathrm{AL}-\mathrm{CH}(0.5 \%)$

(b)

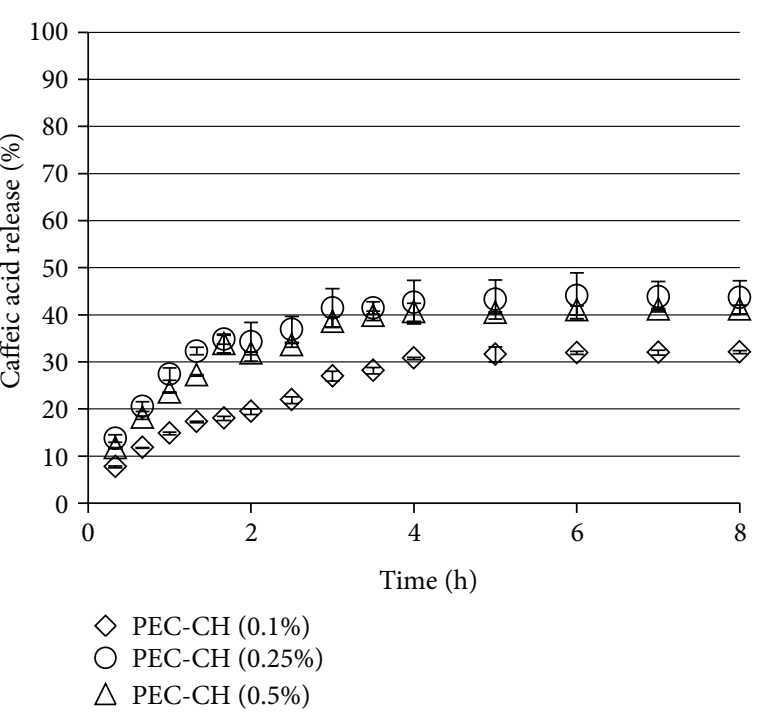

(c)

FIgUre 2: Caffeic acid release profile of SLN (a), coated AL-CH (b), and PEC-CH (c) SLN in gastrointestinal conditions.

caffeic acid release for the three applied concentrations. The release in gastric solution from AL-CH was found to be delayed due to cross-linking of guluronic acids with the $\mathrm{Ca}^{+2}$ ions and the amino groups of chitosan. On the other hand, in the intestinal condition, the slower release could be attributed to the insoluble chitosan blended in the gel structure. PEC-CH coated carriers in pectin concentration of $0.1 \%$ were found to be the most efficient against caffeic acid release in gastric media ( $19.43 \pm 0.59 \%$ of caffeic acid release) (Figure 2(c)). At higher concentrations, it seems that pectin could not properly cover the particle surface and therefore was not capable of decelerating caffeic acid release in gastric media. The micron size of pectin coated carriers in concentrations of 0.25 and $0.50 \%$ shows that additional pectin tends to be aggregated and failed to completely cover the particle surface. Pectin can form a rigid gels by the action of calcium ions cross-linking the galacturonic acid chains [34].

It is also said to resist the proteases and amylase which are active in the upper gastrointestinal tract. 


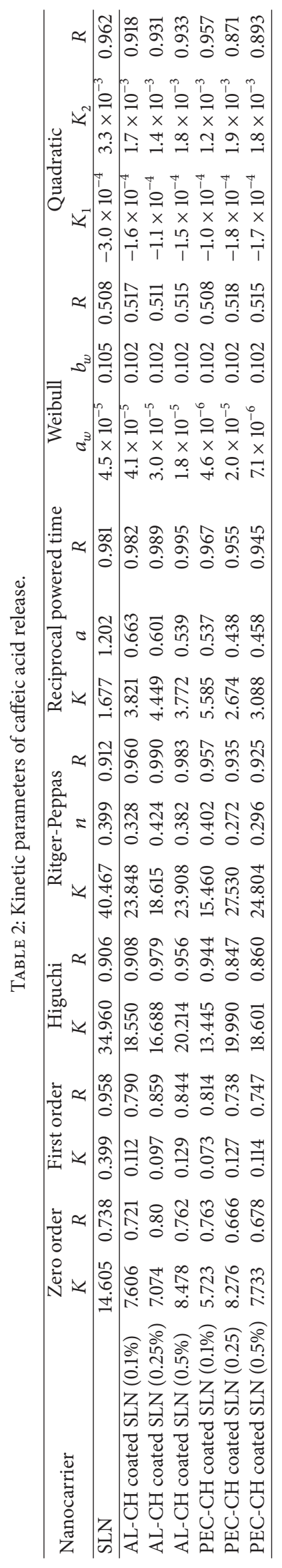


TABLE 3: Antioxidant activity changes ${ }^{\mathrm{a}}$ of released caffeic acid from nanocarriers in gastric and intestinal simulated condition.

\begin{tabular}{lcccc}
\hline \multirow{2}{*}{ Treatment } & \multicolumn{2}{c}{ DPPH assay $(\%)$} & \multicolumn{2}{c}{ Reducing activity $(\mu \mathrm{g} / \mathrm{mL}$ of caffeic acid) } \\
Intestine media
\end{tabular}

T1: caffeic acid released from solid lipid nanoparticles.

T2: caffeic acid released from solid lipid nanoparticles coated with $0.1 \%$ PEC-CH.

T3: caffeic acid released from solid lipid nanoparticles coated with $0.25 \%$ PEC-CH.

T4: caffeic acid released from solid lipid nanoparticles coated with $0.5 \% \mathrm{PEC}-\mathrm{CH}$.

T5: caffeic acid released from solid lipid nanoparticles coated with $0.1 \% \mathrm{AL}-\mathrm{CH}$.

T6: caffeic acid released from solid lipid nanoparticles coated with $0.25 \% \mathrm{AL}-\mathrm{CH}$.

T7: caffeic acid released from solid lipid nanoparticles coated with $0.5 \% \mathrm{AL}-\mathrm{CH}$.

${ }^{\mathrm{a}}$ Results are expressed as the means of three replications \pm standard error of mean.

The modeling results for four different kinetic models are tabulated in Table 2.

Rigter-Peppas and reciprocal powered time models provided better capability to describe release behavior of caffeic acid. The poor ability of zero-order model demonstrated that the release mechanism was concentration dependent. Low correlation coefficients of Higuchi model for pectin coated SLN in concentrations of $0.25 \%$ and $0.5 \%$ could be attributed to the swelling nature of these nanocarriers and weakness of this model for swellable polymers. Rigter-Peppas was found to be the most appropriate model describing release of hesperetin from coated nanostructure lipid carriers and Higuchi the least appropriate model due to swellability of biopolymers [8]. The $n$ values of the Rigter-Peppas model were found to be lower than 0.43 indicating that the release mechanism is governed by the Fickian mechanism. Kinetic study of Weibull model demonstrated that $a_{w}$ values are lower than 1 which indicated a parabolic release curve with a higher initial slope and then a consistent exponential release. These results showed that although burst release was decreased by carbohydrate coating, however, initial release is still exist.

3.3. Antioxidant Activity. The results of antioxidant properties of released caffeic acid in the gastric and intestine solutions for different treatments measured by a couple of distinct experimental methods are presented in Table 3.

Among different treatments, the highest antioxidant activity of the released caffeic acid in the intestine solution obtained for the trial contained noncoated particles (T1) was in line with the highest concentration of caffeic acid excreted in intestine simulated buffer in the given treatment. Instead, in the former phase, in the gastric solution, the measured antioxidant activity in each trial did not represent the trend observed in the caffeic acid release. It seems that the determined antioxidant activity was influenced by the nature of the coated material used in this study. Based on a previous study, pure caffeic acid is an effective DPPHfree radical scavenger and showed appropriate total reducing power [35]. In accordance with the results of PEC-CH (0.1\%) coated treatment in retarding caffeic acid diffusion in gastric solution, antioxidant activity was shown to be lower in this treatment than in noncoated nanocarriers (control). Though, the given treatment did not give rise to the enhancement of this property in the latter step as well. It is noteworthy that the results obtained from reducing activity method were concentration dependent, and therefore, their comparison for different release values might be imprecise.

The results was also shown to be highly influenced by the $\mathrm{pH}$ of the media; thus, in the present study, two individual conditions were considered for assessing the DPPHfree radicals scavenging capability of caffeic acid in different gastrointestinal conditions that unlikely contributes the test's precision. In vitro liberation of encapsulated phenolic component bioactive has been shown to be $\mathrm{pH}$ dependent and acidic environment accelerate the diffusion of protected bioactive [36].

Antioxidant property is defined as the ability of compounds to inhibit or suppress the oxidation process by scavenging the free radicals and/or reactive oxygen species. Methods to determine overall antioxidant activities are divided into two major categories; (i) methods in which the measurement is carried out based on transition of one electron from an antioxidant molecule to an oxidizing agent and (ii) methods in which the hydrogen releasing power of antioxidant molecule is regarded as the antioxidant capability [37]. In the present study, both methods were applied; however, the negative charge on the surface of coated nanoparticles (pectin and alginate) may interfere with the hydrogen donation properties of caffeic acid, leading to sophisticated results.

Based on previous studies, examining a distinct food sample by different antioxidative evaluation methods does not necessarily end up to similar results, and different experiments may result in different responses in quantity and even quality of antioxidant characteristic of a tested sample [38]. Here, in this study, such difference was seen 
between DPPH assay and reducing activity test for both kinds of media, particularly in the gastric buffer, convening that the methodology for evaluating antioxidative characteristics of these structures need to be optimized in future studies. The necessity of application of modified DPPH assay for the determination of antioxidant properties of nanoparticles was also stated by others [39]. Using polymeric nanoparticles of L, D lactid-co-glycolid, Pool et al. [36] showed that encapsulation of antioxidant biochemical like quercetin and catechin resulted in the higher antioxidant capability than their free forms. Inhibition of superoxide production and chelating the prooxidant ions were used in their study as the antioxidative indexes; however, the corresponded protocols were adjusted upon the tests condition.

\section{Conclusions}

In this study, development and release kinetic modeling of novel nanocarriers encasing caffeic acid were pursued. Solid lipid nanoparticles were first used for encapsulation of caffeic acid, while its burst release and low zeta potential made them inappropriate carriers for colon delivery. Layer by layer coating of SLN was therefore performed using alginate-chitosan and pectin-chitosan in different concentrations. Micron size carriers were constructed in higher concentrations $(0.25$ and $0.5 \%$ ) of pectin-chitosan treatments, and nanosize particles with capability of control release were achieved using alginate-chitosan and $0.1 \%$ pectin-chitosan samples. However, the antioxidant properties of the produced nanocarriers in the intestine simulated buffer were not improved by any of the employed treatments which may root by the nature of the coating material. Since bioavailability of nutraceuticals is improved by their colon delivery, developed nanocarriers could be applied for the production of functional fortified food.

\section{References}

[1] F. Shahidi and M. Naczk, Food Phenolics: Sources, Chemistry, Effects, Applications, Taylor \& Francis, London, UK, 2003.

[2] S. Ou and K. Kwok, "Ferulic acid: pharmaceutical functions, preparation and applications in foods," Journal of the Science of Food and Agriculture, vol. 84, no. 11, pp. 1261-1269, 2004.

[3] A. Saija, A. Tomaino, R. Lo Cascio et al., "Ferulic and caffeic acids as potential protective agents against photooxidative skin damage," Journal of the Science of Food and Agriculture, vol. 79, no. 3, pp. 476-480, 1999.

[4] M. Fathi, M. R. Mozafari, and M. Mohebbi, "Nanoencapsulation of food ingredients using lipid based delivery systems," Trends in Food Science and Technology, vol. 23, no. 1, pp. 13-27, 2012.

[5] W. Klaypradit and Y. Huang, "Fish oil encapsulation with chitosan using ultrasonic atomizer," LWT-Food Science and Technology, vol. 41, no. 6, pp. 1133-1139, 2008.

[6] M. Fathi, J. Varshosaz, M. Mohebbi, and F. Shahidi, "Hesperetinloaded solid lipid nanoparticles and nanostructure lipid carriers for food fortification: preparation, characterization, and modeling," Food and Bioprocess Technology, vol. 6, no. 6, pp. 14641475, 2013.
[7] G. Y. Park, S. Mun, Y. Park et al., "Influence of encapsulation of emulsified lipids with chitosan on their in vivo digestibility," Food Chemistry, vol. 104, no. 2, pp. 761-767, 2007.

[8] M. Fathi and J. Varshosaz, "Novel hesperetin loaded nanocarriers for food fortification: production and characterization," Journal of Functional Foods, vol. 53, pp. 1382-1391, 2013.

[9] R. Hejazi and M. Amiji, "Chitosan-based gastrointestinal delivery systems," Journal of Controlled Release, vol. 89, no. 2, pp. 151165, 2003.

[10] T. Espevik, M. Otterlei, G. Skjak-Braek, L. Ryan, S. D. Wright, and A. Sundan, "The involvement of CD14 in stimulation of cytokine production by uronic acid polymers," European Journal of Immunology, vol. 23, no. 1, pp. 255-261, 1993.

[11] W. R. Gombotz and S. F. Wee, "Protein release from alginate matrices," Advanced Drug Delivery Reviews, vol. 31, no. 3, pp. 267-285, 1998.

[12] M. George and T. E. Abraham, "Polyionic hydrocolloids for the intestinal delivery of protein drugs: alginate and chitosana review," Journal of Controlled Release, vol. 114, no. 1, pp. 1-14, 2006.

[13] S. Sugawara, T. Imai, and M. Otagiri, "The controlled release of prednisolone using alginate gel," Pharmaceutical Research, vol. 11, no. 2, pp. 272-277, 1994.

[14] C.-K. Kim and E.-J. Lee, "The controlled release of blue dextran from alginate beads," International Journal of Pharmaceutics, vol. 79, no. 1, pp. 11-19, 1992.

[15] R. H. Walter and S. Taylor, The Chemistry and Technology of Pectin, Elsevier Science, Amsterdam, The Netherlands, 1992.

[16] R. A. Nash and B. E. Haeger, "Zeta potential in the development of pharmaceutical suspensions," Journal of Pharmaceutical Sciences, vol. 55, no. 8, pp. 829-837, 1966.

[17] G. Singhvi and M. Singh, "Review: in-vitro drug release characterization models," International Journal of Pharmaceutical Studies and Research, vol. 2, no. 1, pp. 77-84, 2011.

[18] P. Costa and J. M. Sousa Lobo, "Modeling and comparison of dissolution profiles," European Journal of Pharmaceutical Sciences, vol. 13, no. 2, pp. 123-133, 2001.

[19] M. Chakraborty, S. Dasgupta, C. Soundrapandian et al., "Methotrexate intercalated ZnAl-layered double hydroxide," Journal of Solid State Chemistry, vol. 184, no. 9, pp. 2439-2445, 2011.

[20] C. R. Rekha and G. Vijayalakshmi, "Biomolecules and nutritional quality of soymilk fermented with probiotic yeast and bacteria," Applied Biochemistry and Biotechnology, vol. 151, no. 2-3, pp. 452-463, 2008.

[21] A. N. Shinde, N. Malpathak, and D. P. Fulzele, "Determination of isoflavone content and antioxidant activity in Psoralea corylifolia L. callus cultures," Food Chemistry, vol. 118, no. 1, pp. 128-132, 2010.

[22] E. Zimmermann and R. H. Müller, "Electrolyte- and pHstabilities of aqueous solid lipid nanoparticle (SLN) dispersions in artificial gastrointestinal media," European Journal of Pharmaceutics and Biopharmaceutics, vol. 52, no. 2, pp. 203-210, 2001.

[23] A. Hentschel, S. Gramdorf, R. H. Müller, and T. Kurz, “ $\beta$ Carotene-loaded nanostructured lipid carriers," Journal of Food Science, vol. 73, no. 2, pp. N1-N6, 2008.

[24] E. Gonzalez-Mira, M. A. Egea, M. L. Garcia, and E. B. Souto, "Design and ocular tolerance of flurbiprofen loaded ultrasoundengineered NLC," Colloids and Surfaces B, vol. 81, no. 2, pp. 412$421,2010$. 
[25] P. Severino, M. H. A. Santana, and E. B. Souto, "Optimizing SLN and NLC by 22 full factorial design: effect of homogenization technique," Materials Science and Engineering C, vol. 32, no. 6, pp. 1375-1379, 2012.

[26] C.-C. Chen, T. Tsai, Z. Huang, and J. Fang, "Effects of lipophilic emulsifiers on the oral administration of lovastatin from nanostructured lipid carriers: physicochemical characterization and pharmacokinetics," European Journal of Pharmaceutics and Biopharmaceutics, vol. 74, no. 3, pp. 474-482, 2010.

[27] T. Li, X. Shi, Y. Du, and Y. Tang, "Quaternized chitosan/alginate nanoparticles for protein delivery," Journal of Biomedical Materials Research A, vol. 83, no. 2, pp. 383-390, 2007.

[28] K. Mladenovska, R. S. Raicki, E. I. Janevik et al., "Colon-specific delivery of 5-aminosalicylic acid from chitosan-Ca-alginate microparticles," International Journal of Pharmaceutics, vol. 342, no. 1-2, pp. 124-136, 2007.

[29] K. Mladenovska, O. Cruaud, P. Richomme et al., “5-ASA loaded chitosan-Ca-alginate microparticles: preparation and physicochemical characterization," International Journal of Pharmaceutics, vol. 345, no. 1-2, pp. 59-69, 2007.

[30] J. R. R. de Souza, J. I. X. de Carvalho, M. T. S. Trevisan, R. C. M. de Paula, N. M. P. S. Ricardo, and J. P. A. Feitosa, "Chitosancoated pectin beads: characterization and in vitro release of mangiferin," Food Hydrocolloids, vol. 23, no. 8, pp. 2278-2286, 2009.

[31] J.-S. Lee, J. S. Kim, and H. G. Lee, “ $\gamma$-Oryzanol-loaded calcium pectinate microparticles reinforced with chitosan: optimization and release characteristics," Colloids and Surfaces B, vol. 70, no. 2, pp. 213-217, 2009.

[32] M. Tholstrup Sejersen, T. Salomonsen, R. Ipsen, R. Clark, C. Rolin, and S. Balling Engelsen, "Zeta potential of pectinstabilised casein aggregates in acidified milk drinks," International Dairy Journal, vol. 17, no. 4, pp. 302-307, 2007.

[33] Q. Luo, J. Zhao, X. Zhang, and W. Pan, "Nanostructured lipid carrier (NLC) coated with Chitosan Oligosaccharides and its potential use in ocular drug delivery system," International Journal of Pharmaceutics, vol. 403, no. 1-2, pp. 185-191, 2011.

[34] P. Sriamornsak, S. Sungthongjeen, and S. Puttipipatkhachorn, "Use of pectin as a carrier for intragastric floating drug delivery: carbonate salt contained beads," Carbohydrate Polymers, vol. 67, no. 3, pp. 436-445, 2007.

[35] İ. Gülçin, "Antioxidant activity of caffeic acid (3,4-dihydroxycinnamic acid)," Toxicology, vol. 217, no. 2-3, pp. 213-220, 2006.

[36] H. Pool, D. Quintanar, J. de Dios Figueroa et al., "Antioxidant effects of quercetin and catechin encapsulated into PLGA nanoparticles," Journal of Nanomaterials, vol. 2012, Article ID 145380, 12 pages, 2012.

[37] O. I. Aruoma and S. L. Cuppett, Antioxidant Methodology: In Vivo and In Vitro Concepts, AOCS Press, Urbana, Ill, USA, 1997.

[38] Y.-C. Wang, R.-C. Yu, and C.-C. Chou, "Antioxidative activities of soymilk fermented with lactic acid bacteria and bifidobacteria," Food Microbiology, vol. 23, no. 2, pp. 128-135, 2006.

[39] S. Barnaby, N. Sarker, A. Dowdell, and I. Banerjee, "The spontaneous formation of selenium nanoparticles on gallic acid assemblies and their antioxidant properties," The Fordham Undergraduate Research Journal, vol. 1, pp. 41-46, 2011. 

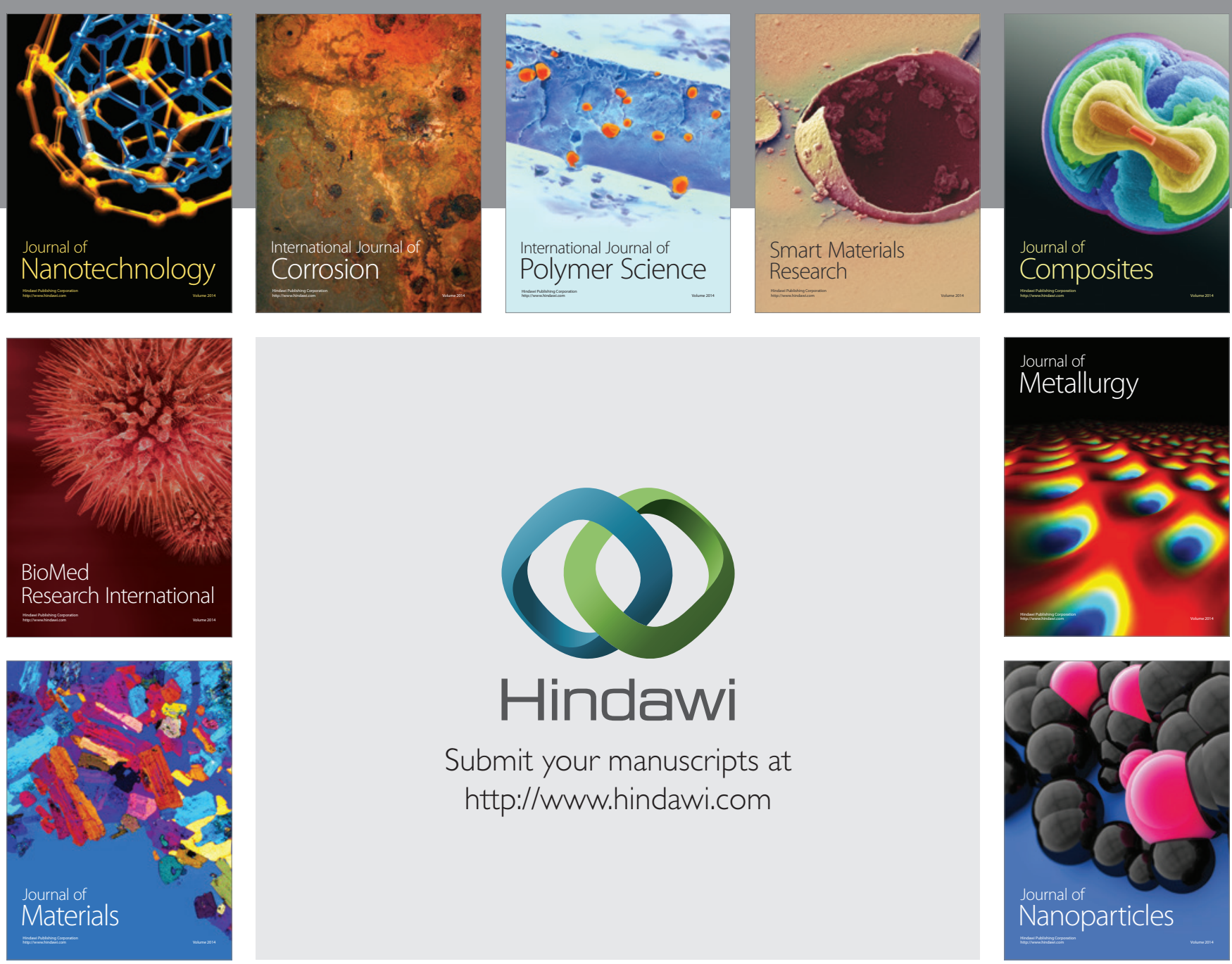

Submit your manuscripts at http://www.hindawi.com
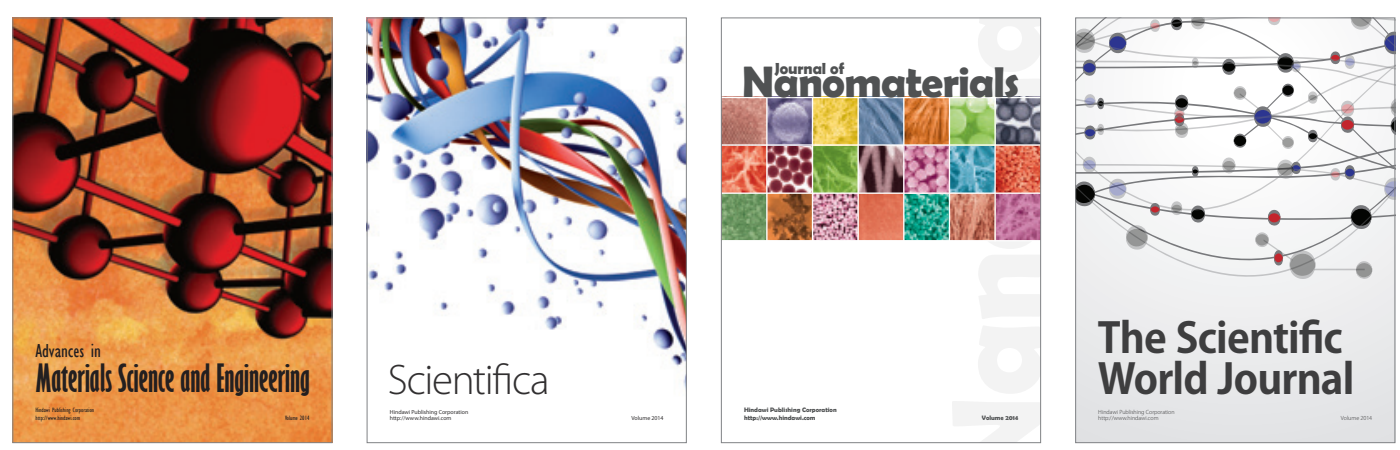

\section{The Scientific World Journal}
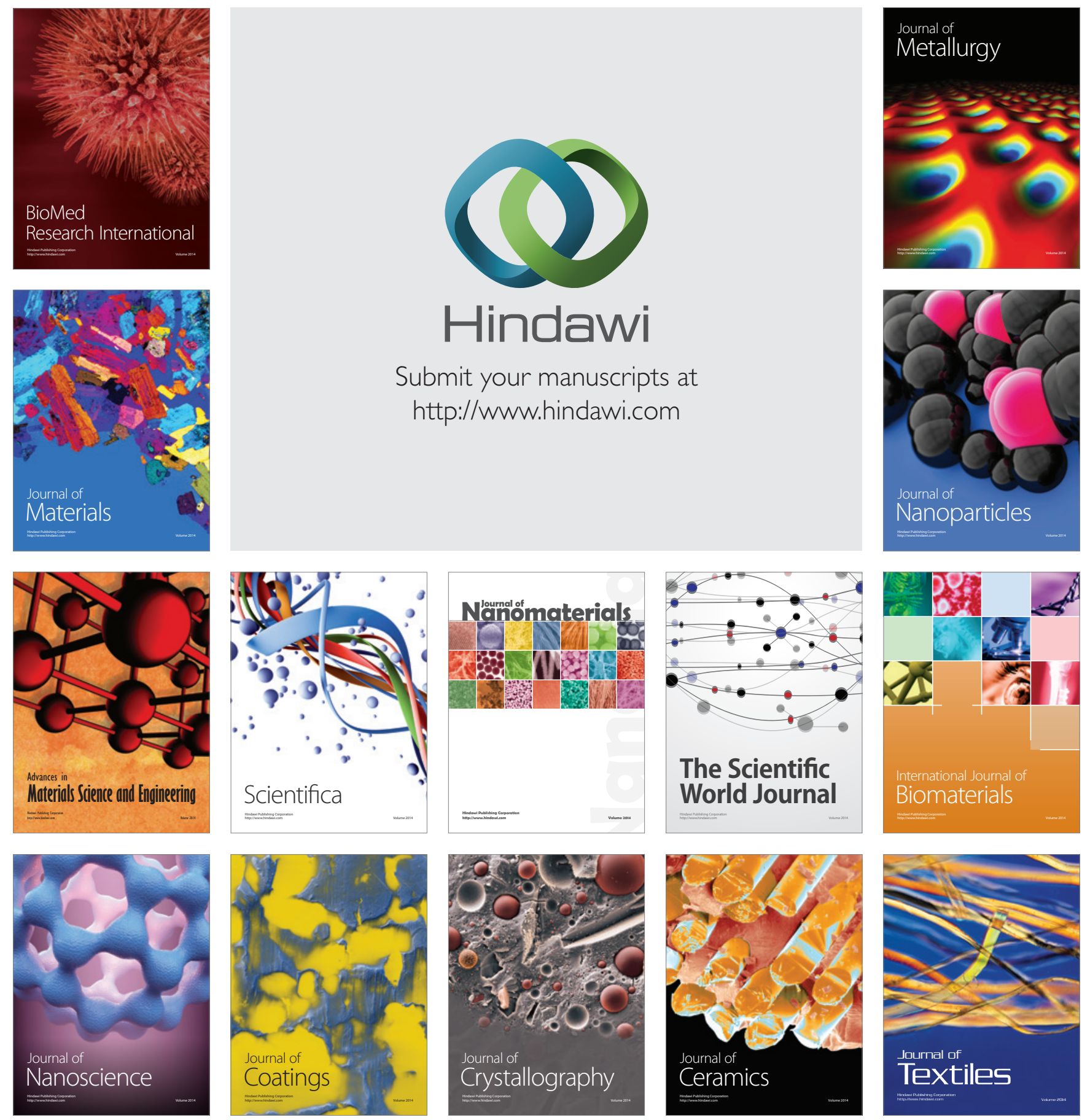